\title{
Development of new flow stabilizers for increasing the reliability and efficiency of power equipment
}

\author{
A. Rogalev, A. Kocherova, I. Komarov, I. Garanin \\ \& G. Kurdiukova \\ Moscow Power Engineering Institute, Russia
}

\begin{abstract}
The paper explores the nature and the cause of dynamic forces exerted by the flow on the walls of energy equipment. The most loaded parts are the ones in which there is a sharp change of the working medium flow direction (steam supply system of a turbine, valves, pipeline bends). In these parts, centrifugal forces cause a transverse pressure gradient, which leads to separations of the flow. The separations cause broad frequency spectrum pressure oscillations, the amplitude of those may reach more than $10 \%$ of the initial pressure. As a result, energy equipment has to work under a heavy dynamic load.

There are some effective ways to reduce dynamic loads, and the most popular of them is the installation of a special device - a flow stabilizer - in the parts of equipment that suffer from them. The existing stabilizers are mostly the disk with different kinds of perforation, which are installed transversely to the flow direction. They dispel the separation vortexes and level uneven velocity profiles. It is obvious that they have such a high hydraulic resistance that it can nullify their economic effect. The resistance is mostly reduced by enlargement of the stabilizer's diameter that causes some designing and exploitation difficulties.

In this paper some new designs of flow stabilizers are proposed. The most part of the new device's surface is located streamwise, so they have the diameter of the pipeline. The comparative estimation revealed that the newly developed devices provide a comparable level of dynamic load reduction, but also have a much lower hydraulic resistance ( 3 and more times less) and are supposed to have a higher mechanical strength.
\end{abstract}

Keywords: power plant equipment, steam distribution system of steam turbines, non-uniform velocity profile, dynamic loads, flow stabilizer. 


\section{Introduction}

Analysis of failures of electric power plants and their technologically dependent facilities shows that equipment - such as thermomechanical machinery, valves and pipelines - is most often damaged by dynamic loads impacting its components from within the process medium. Loads are maximized in locations where the process medium flow changes its direction abruptly. This is explained by the action of centrifugal forces that throw liquid particles toward outer (concave) walls of curved duct, bringing about a transverse pressure gradient [1].

This further promotes the development of detached flows ultimately manifesting themselves an uneven flow velocity profile. In turn, the non-uniform and non-stationary behavior of the velocity field means even greater unevenness of pressure distribution within the flow. Thus, the task of improving reliability of power plant equipment calls for a detailed study of the nature of dynamic loads and their originative flow structure, to devise efficient countermeasures.

\section{Properties of dynamic loads acting from within the flow in a curved duct}

As they act on duct walls, time-variable pressure forces generate vibrations in a wide frequency range. Experimental studies of pressure pulsations in steamcarrying parts of a turbine unit with initial rated parameters of $24 \mathrm{MPa} ; 540^{\circ} \mathrm{C}$ have revealed low-frequency vibrations $(3.3 ; 7.5 ; 10.5 \mathrm{~Hz})$ with an average amplitude of $2.5 \mathrm{MPa}$ in various modes, reaching 4 to $5 \mathrm{MPa}$ in individual cases. Within the spectral range of 400 to $450 \mathrm{~Hz}$, vibration amplitudes reach 2.3 to 2.5 $\mathrm{MPa}$, and at higher frequencies (for example, up to $1150 \mathrm{~Hz}$ ), while the amplitude stays within 1.0 to $1.2 \mathrm{MPa}$, vibration energy may be significantly higher due to increased frequency of vibration [2].

Obviously, such pressure fluctuations are severely detrimental to equipment reliability. The search for an effective remedy would call first of all for looking at the flow pattern that brings about dynamic loads so sharp. Numerical simulation (CFD modeling) is the most rational approach to this problem.

Findings from flow simulation in a curved duct reproducing a part of turbine unit steam transfer system (fig. 1) point to the formation of detached vortices in curved parts of the duct sufficiently powerful to disturb long pipeline sections. Computations assumed isothermal flows with adiabatic pipeline walls ( $D=100 \mathrm{~mm}$ ), outlet pressure equal to atmospheric pressure, and flows dimensioned so that mean velocity would average $60 \mathrm{~m} / \mathrm{s}$ - representative of power plant equipment.

The flow velocity profile measured immediately after the turn similarly points to the presence of detached flows together with a significant non-uniformity of the pressure field. A velocity profile for a uniform flow in a straight pipe at the same mass flow was shown for comparison.

It is known that the frequency of pressure fluctuations is directly linked with the size of vortical formations: the greater are the vortices, the lower is the fluctuation frequency and the higher is the energy potential of fluctuating motion. 
Large areas of vortical currents are the cause for high-amplitude vibrations in turbine's steam supply system described earlier. As vortex sizes diminish, their velocity within the flow increases to match the velocity of the flow itself, the resulting fluctuations become more frequent and the total energy of vortical motion declines sharply. Thus, the main approach for decreasing dynamic loads on duct walls lies with breaking large vortex kernels into smaller formations and distributing them uniformly across the duct $[3,4]$.
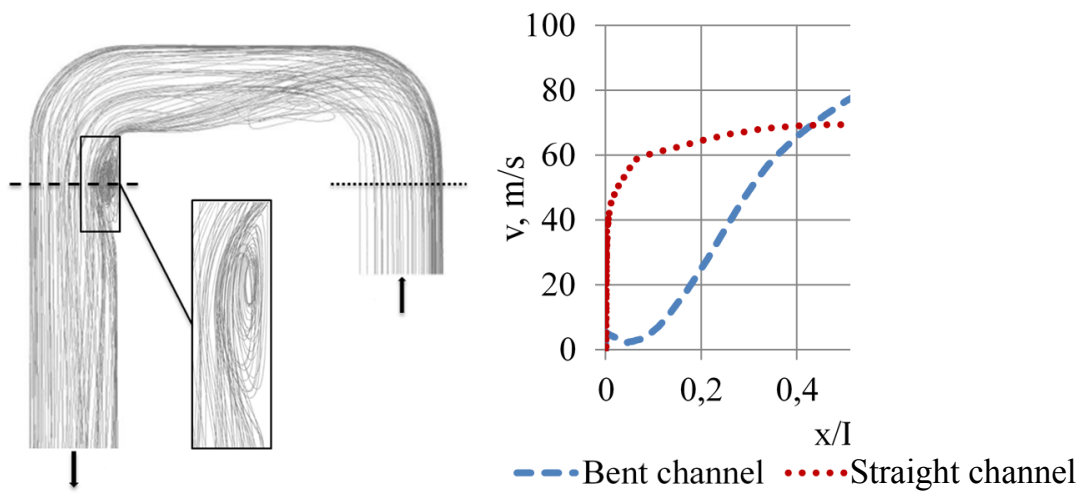

Figure 1: Flow in a curved part of a steam supply duct of a steam turbine.

\section{Existing flow stabilizer designs}

The pressure fluctuation suppression principle described above is implemented using so-called flow stabilizers (aerodynamic filters, vortex suppressors). Most modern designs comprise a variety of perforated disc-shaped structures installed in the pipeline. Table 1 summarizes structural designs of the most common stabilizers and their action on non-uniform flow downstream of the abovementioned curved member of steam supply duct in a steam turbine. On average they are capable of reducing pressure fluctuation amplitude almost three-fold, with vibrational motion becoming 2.2 times lower [5]. Findings from CFD computations for such stabilizer design are shown in fig. 2. The non-uniformity is similarly introduced by the above-described curvilinear steam supply duct member in a steam turbine (fig. 3) and boundary conditions are the same.

If, as fig. 1 shows, the vortex occupied above $40 \%$ of pipeline cross-section area before the stabilizer was installed, and vortex-induced vibrations took at least five pipeline gauges to fade out, vortices are suppressed virtually instantly now - at a distance less than half of pipeline gauge.

However, the introduction of a flow stabilizer causes hydraulic resistance of the system to increase significantly as the free section area diminishes. Discshaped stabilizers feature hydraulic resistance factors of 9 and higher, often making their use inexpedient despite all the benefits [6]. Thus, it is of great relevance to design flow stabilizers that, in addition to reducing dynamic loads on equipment components, would possess low hydraulic resistance. 


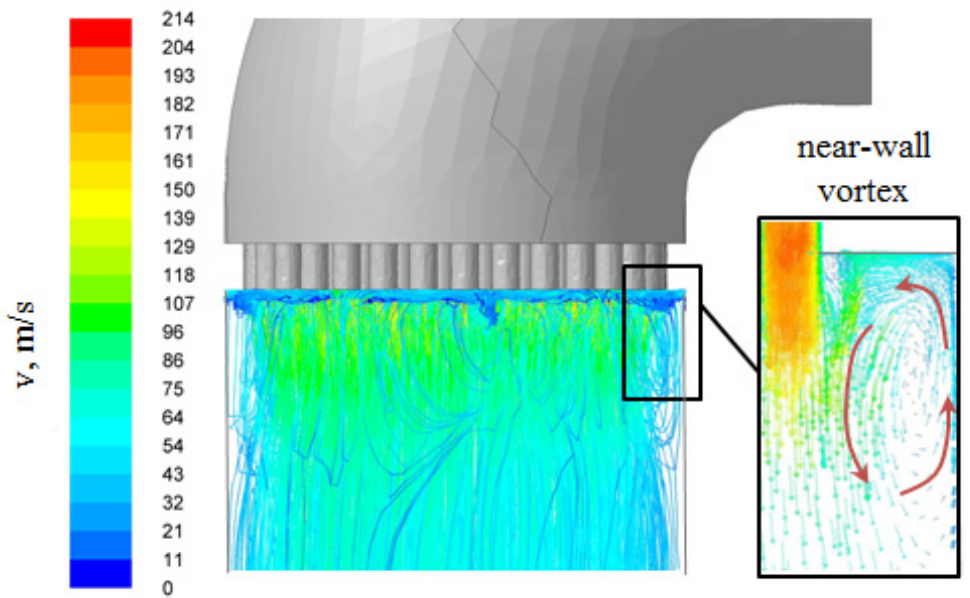

Figure 2: Results of a perforated disc-shaped flow stabilizer CFD-simulation.

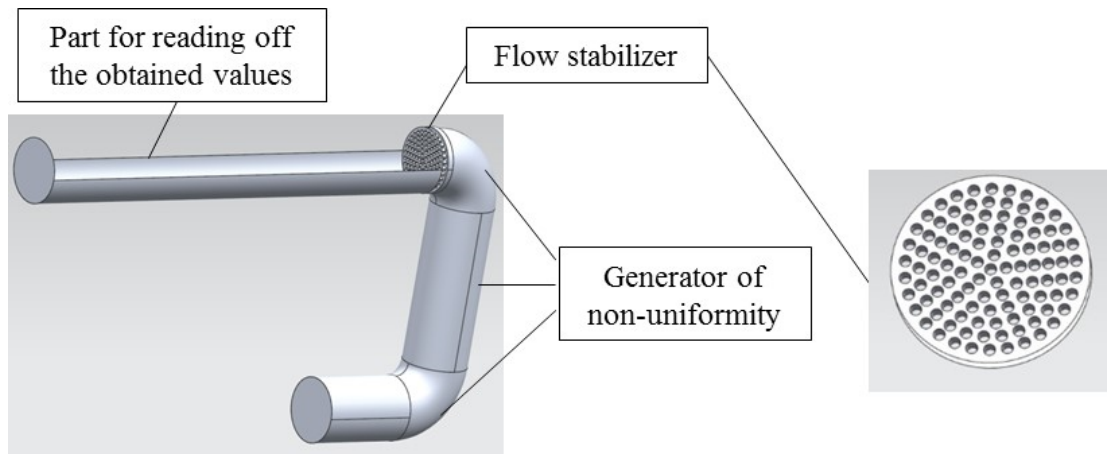

Figure 3: Sample numerical simulation model of a perforated disc-shaped flow stabilizer.

\section{New flow stabilizer concept}

Meeting the above-mentioned performance objective requires first of all to increase the free flow section of the device. The most beneficial approach in this case would involve reducing the lateral surface area of the stabilizer (across the flow direction) as vortices can still be broken effectively by promoting interaction of the medium with thin surfaces placed at short intervals along the flow. Hydraulic resistance will be further reduced by the use of perforated 
Table 1: Common types of flow stabilizers.

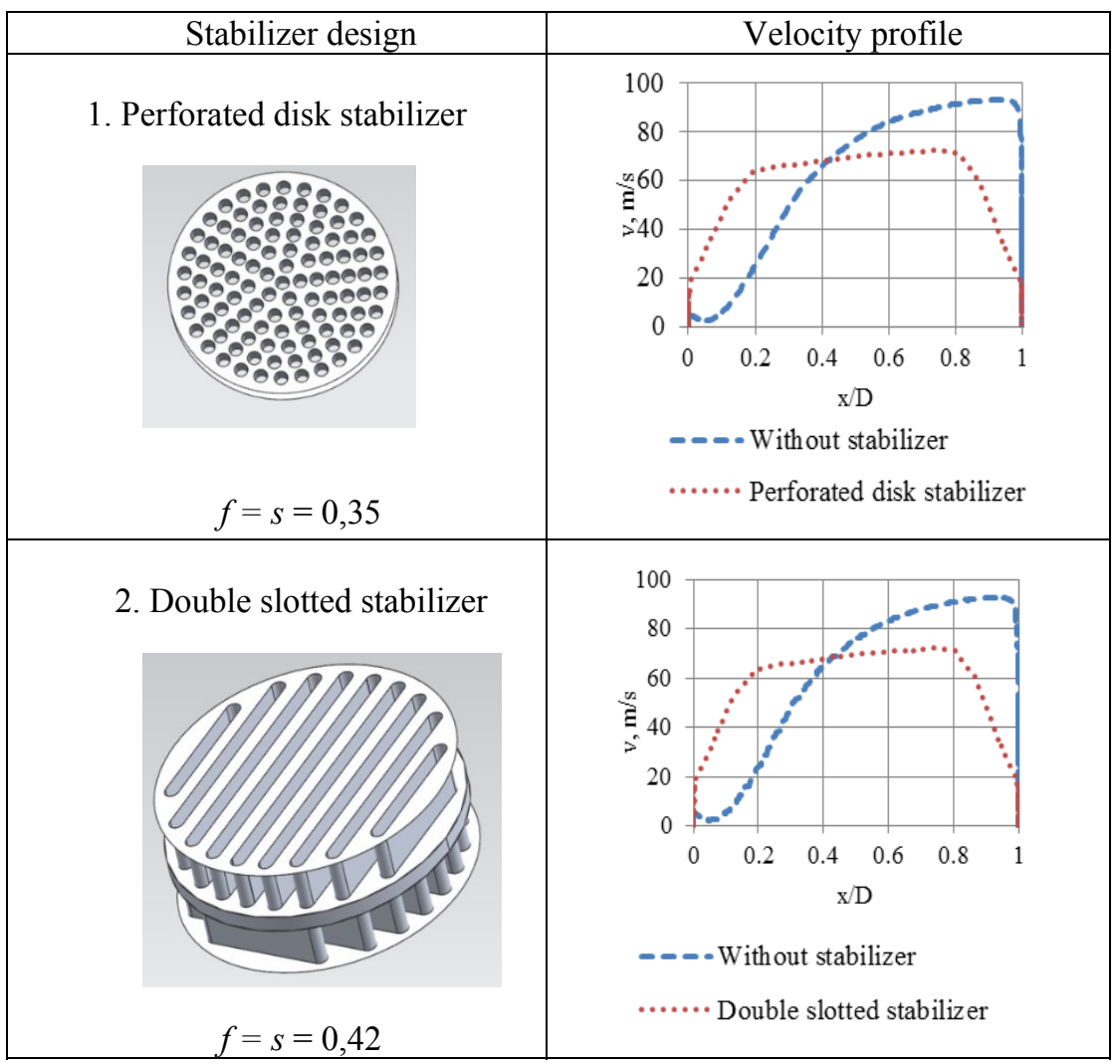

stabilizer surfaces. In addition to letting the liquid to flow freely under the action of lateral pressure gradient caused by flow rotation, such a design also decreases the pressure drop acting on device components laterally, thereby improving reliability of the device.

Researchers from the Moscow Power Engineering Institute used these considerations to come up with several new designs of flow stabilizers (table 2).

Key performance parameters of flow stabilizers include:

- surface perforation factor defined as the area of holes to the area of a similar unperforated surface:

$$
f=\frac{F_{\text {hole }}}{F_{\Sigma}}
$$

- free flow factor, defined as a ratio of free-flow section areas before and after installing the stabilizer:

$$
S=\frac{S_{\text {stab }}}{S_{\text {orig }}}
$$


Table 2: Developed flow stabilizers.

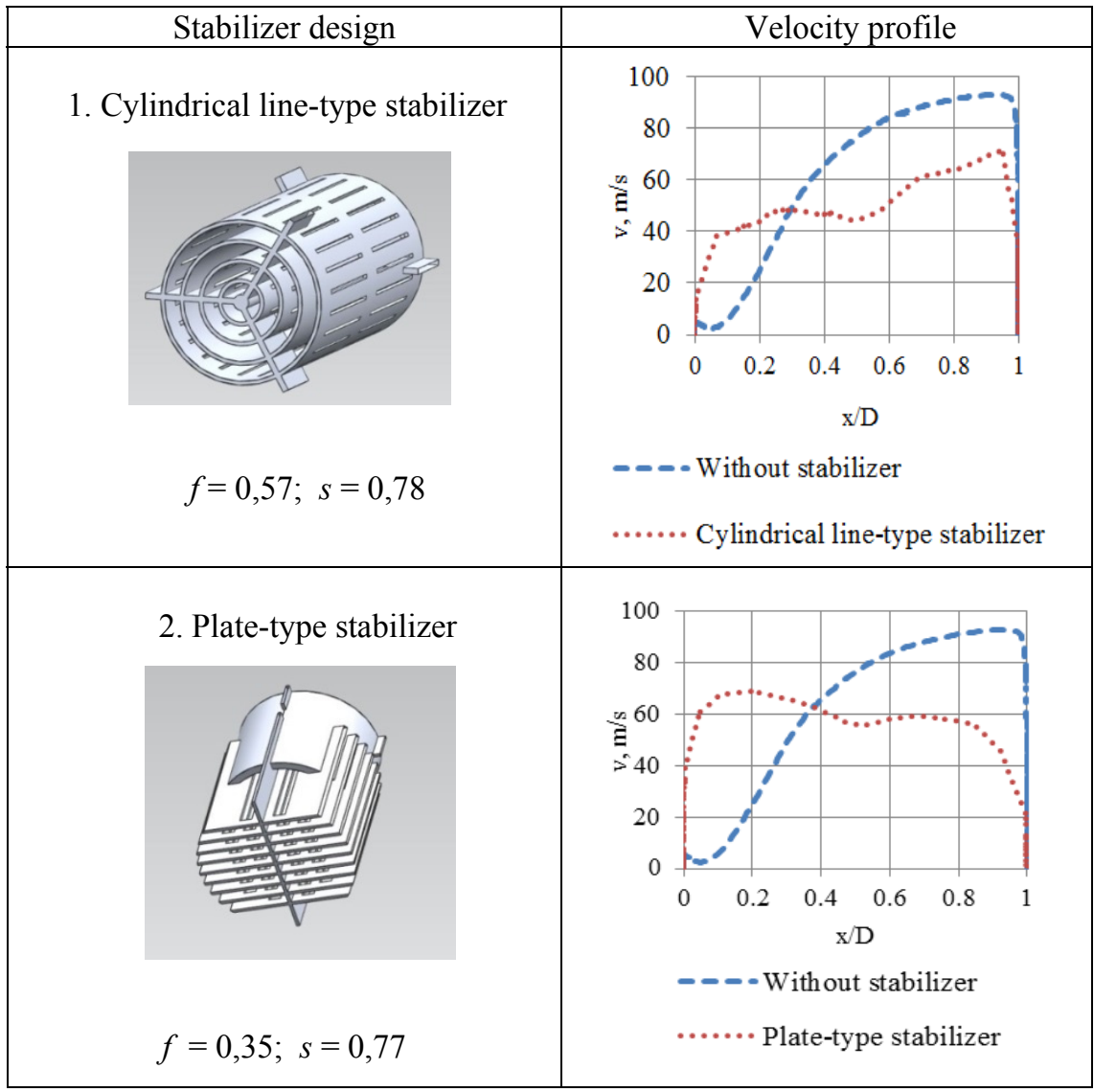

This factor is introduced to describe new stabilizer designs that have their primary working surface situated along the flow in the pipeline, therefore the perforation factor alone does not provide full information on the free-flow section area. This option involved computations for a pipeline of $100 \mathrm{~mm}$ internal diameter, however, as long as the same design parameters are met, similar stabilizers may be designed for pipelines of any diameter.

\section{Estimating the efficiency of flow stabilizers}

Velocity profile irregularity in CFD simulations is indicative of the intensity of dynamic loads acting on equipment walls. Consequently, a quantitative estimate is needed to assess flow stabilizer efficiency.

To that end, an index of non-uniformity is used, determined as follows [7]:

- index of non-uniformity for $i$-th point in the velocity profile: 


$$
\omega_{i}=\frac{\left|u_{i}-\bar{u}\right|}{\bar{u}}
$$

where $u_{i}$ is the velocity in $i$-th point of the profile, $i=1 \ldots n ; \bar{u}$ is the average velocity across the profile.

- index of non-uniformity for the velocity profile:

$$
\omega=\frac{\sum \omega_{i}}{n}
$$

The installation of all flow stabilizer designs described above brings about a significant decline in the index of non-uniformity. This is illustrated clearly in fig. 4: the introduction of a flow stabilizer decreases flow profile non-uniformity - and hence dynamic loads - by $40 \%$.

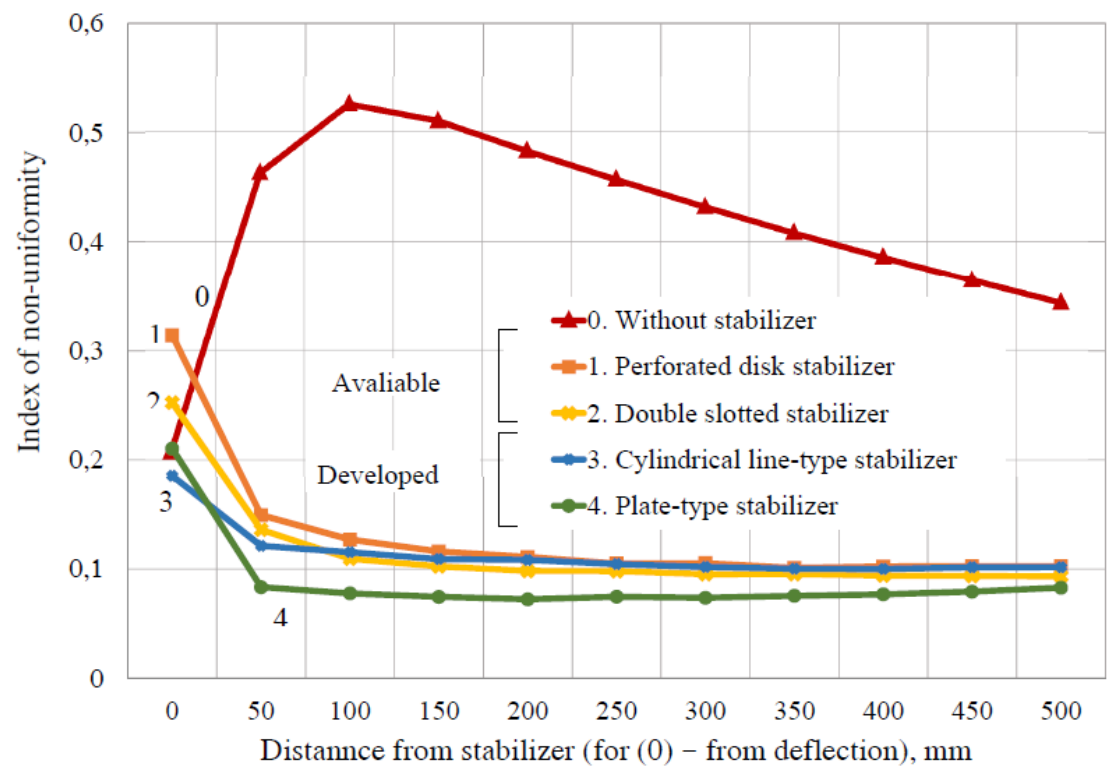

Figure 4: Comparison of flow stabilizers in terms of index of non-uniformity.

The slotted dual stabilizer is the most efficient among the designs used currently. The plate-type stabilizer is the best performer among new devices as illustrated by a plot in fig. 4: its introduction reduces the index of flow nonuniformity by $56 \%$ on average, this decline reaching $65 \%$ just $250 \mathrm{~mm}$ downstream of the stabilizer.

That said, its equalization quality is comparable to that of a dual slotted stabilizer which is also more compact and easier to manufacture.

Nevertheless, the plate-type stabilizer wins in terms of overall efficiency as it features a lower hydraulic resistance (fig. 5). To achieve best performance, this stabilizer must be placed within the pipeline so that plates would be oriented 


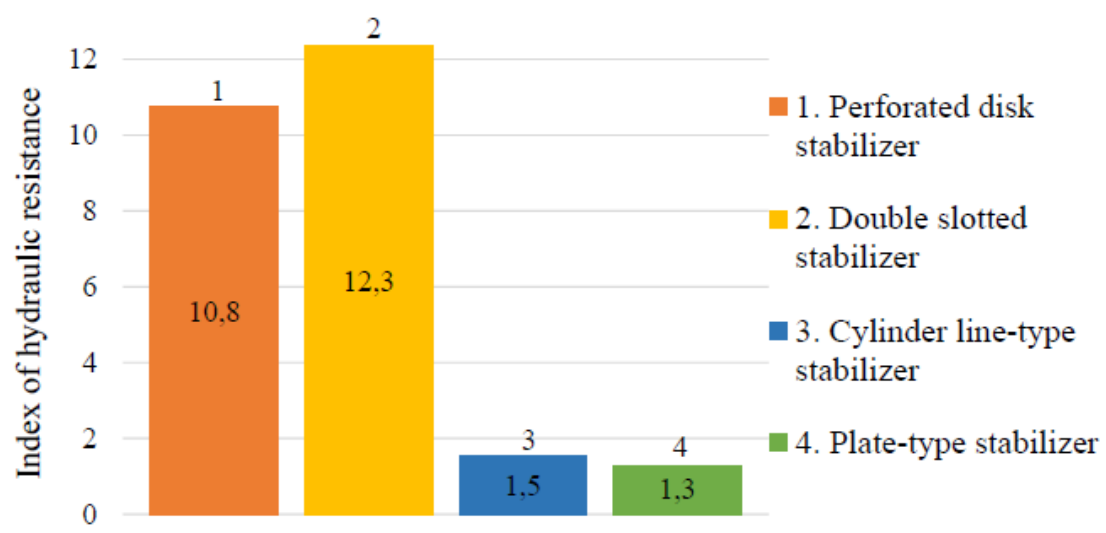

Figure 5: Comparison of flow stabilizers in terms of hydraulic resistance factor.

along the rotation plane, as the lateral pressure gradient that appears when the flow is rotated would act on the thin edge of the plate. In addition to relieving the load on the plate, this solution reduces hydraulic resistance even further.

It should also be noted that at a distance of approximately 3 times the pipeline diameter downstream of the device, the design of the device no longer has any significant bearing on the index of non-uniformity of the flow which remains constant at 0.1 from then on. This is explained by the fact that broken vortices have low energies and dissipate quickly. However, within the preceding section there is a great difference in efficiency among various designs that may have a significant impact on the reliability of a particular system.

Findings from computations of flow in a curved duct enveloping a plate-type stabilizer are shown in fig. 6 . Thin surfaces of the device completely suppress the initial non-uniformity of the flow, preventing the formation of large detached vortices while introducing virtually no new disturbances into the flow. Notable vortex-like formations only appear on the downstream edge of the plate, but they are insignificant and fade out almost instantly.

\section{Conclusions}

A comparison of data shown in fig. 3 and fig. 4 leads to the conclusion that the flow stabilizer design approach described by us brings about a qualitative improvement in the uniformity of flow velocity profile, consequently suppressing dynamic loads acting on equipment walls from within the flow. At the same time, the new design features a hydraulic resistance 3 to 7 times smaller than that of currently used designs. More complex manufacturing process is the only drawback faced by new flow stabilizer designs as compared to currently used options. 


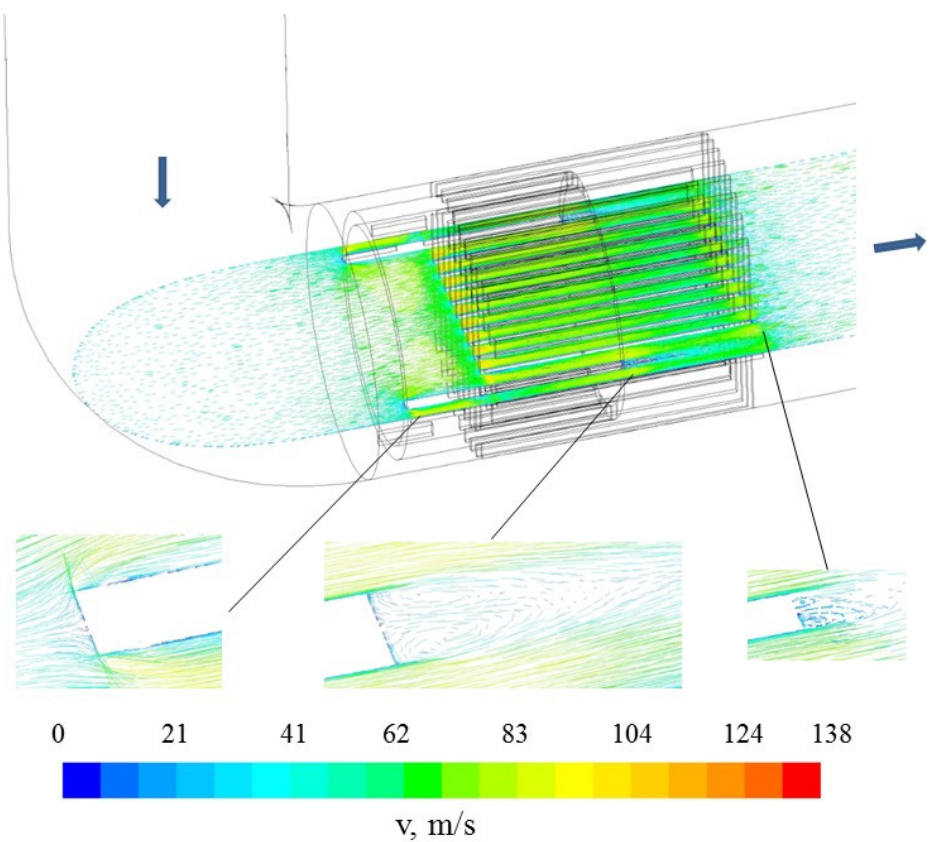

Figure 6: Flow inside channels of a plate-type flow stabilizer (cross-section matching the pipe diameter, $D_{i}=100 \mathrm{~mm}$, plates located in the flow rotation plane).

\section{Acknowledgements}

The research has been carried out in Moscow Power Engineering Institute with financial support from the Russian Federation represented by Ministry of Education and Science of the Russian Federation under Agreement No. 14.574.21.0016 on Grant Provision dated June 17, 2014, for the purpose of implementation of the Federal Target Program "Research and Development in Priority Growth Fields of Russian Science and Technology Sector for 20142020". The unique ID of applied research: RFMEFI57414X0016.

\section{References}

[1] Deitch, M.E. Engineering Gas Dynamics, Gosenergoizdat: Moscow, 1961.

[2] Kostyuk, A.G., Kumenko, A.I., Nekrasov, A.L., Kalinin, S.V., Medvedev S.V. An experimental analysis of pressure pulsations in the steam admission elements of a turbine installation, Thermal Engineering, 6, pp. 529-537, 2000.

[3] Gotovtsev, A.M. Development and research of a system of steam flow stabilization in turbine necks and remote control valves, MPEI: Moscow, 2006. 
484 Energy and Sustainability VI

[4] Zaryankin, V.A. Aerodynamic methods of improving efficiency and reliability of power plants thermal and mechanical equipment elements, MPEI: Moscow, 2000.

[5] Zaryankin, A., Rogalev, N., Rogalev, A., Kocherova, A., Strielkowski, W. Line Summary of Approaches for Improving Reliability of Thermodynamical Equipment and Its Interconnecting Pipelines at Thermal Power Plant, Contemporary Engineering Sciences, 33, pp. 1793-1806, 2014.

[6] Grigoriev, E.Yu. Development and research of vibration reduction methods in annular diffusors of gas turbines, MPEI: Moscow, 2014.

[7] Tsinoglou, D.N, Koltsakis, G.C., Missirlis, D.K, Yakinthos, K.J. Transient modeling of flow distribution in automotive catalytic converters, Applied Mathematical Modelling, 9, pp. 775-794, 2004. 